\title{
Conservation Rules of Direct Sum Decomposition of Groups
}

\author{
Kazuhisa Nakasho \\ Shinshu University \\ Nagano, Japan
}

\author{
Hiroshi Yamazaki \\ Shinshu University \\ Nagano, Japan \\ Yasunari Shidama \\ Shinshu University \\ Nagano, Japan
}

\author{
Hiroyuki Okazaki \\ Shinshu University \\ Nagano, Japan
}

\begin{abstract}
Summary. In this article, conservation rules of the direct sum decomposition of groups are mainly discussed. In the first section, we prepare miscellaneous definitions and theorems for further formalization in Mizar [5]. In the next three sections, we formalized the fact that the property of direct sum decomposition is preserved against the substitutions of the subscript set, flattening of direct sum, and layering of direct sum, respectively. We referred to [14, [13 [6] and 11] in the formalization.
\end{abstract}

MSC: 20E34 03B35

Keywords: group theory; direct sum decomposition

MML identifier: GROUP_21, version: 8.1.04 5.36.1267

\section{PReliminaries}

Let $I, J$ be non empty sets, $a$ be a function from $I$ into $J$, and $F$ be a multiplicative magma family of $J$. Observe that the functor $F \cdot a$ yields a multiplicative magma family of $I$. Let $F$ be a group family of $J$. Let us observe that the functor $F \cdot a$ yields a group family of $I$. Let $G$ be a group and $F$ be a subgroup family of $J$ and $G$. The functor $F \cdot a$ yielding a subgroup family of $I$ and $G$ is defined by the term

(Def. 1) $F \cdot a$. 
The scheme $S$ ch 1 deals with a set $\mathcal{A}$ and a 1 -sorted structure $\mathcal{B}$ and a unary functor $\mathcal{F}$ yielding a set and states that

(Sch. 1) There exists a function $f$ such that $\operatorname{dom} f=\mathcal{A}$ and for every element $x$ of $\mathcal{B}$ such that $x \in \mathcal{A}$ holds $f(x)=\mathcal{F}(x)$.

Let $I$ be a set. Let us note that there exists a many sorted set indexed by $I$ which is non-empty and disjoint valued.

Now we state the propositions:

(1) Let us consider a non-empty, disjoint valued function $f$. If $\bigcup f$ is finite, then $\operatorname{dom} f$ is finite.

Proof: For every objects $x, y$ such that $x, y \in \operatorname{dom} f$ and $f(x)=f(y)$ holds $x=y$ by [7, (3)].

(2) Let us consider non empty sets $X, Y$, sets $X_{0}, Y_{0}$, and a function $f$ from $X$ into $Y$. Suppose $f$ is bijective and $\operatorname{rng}\left(f\left\lceil X_{0}\right)=Y_{0}\right.$. Then $\left(f\left\lceil X_{0}\right)^{-1}=\right.$ $f^{-1}\left\lceil Y_{0}\right.$.

Proof: For every object $x$ such that $x \in \operatorname{dom}\left(f^{-1} \mid Y_{0}\right)$ holds $\left(f^{-1} \mid Y_{0}\right)(x)=$ $\left(f\left\lceil X_{0}\right)^{-1}(x)\right.$ by [18, (62)], [7, (49), (33)], [18, (59)].

\section{Conservation Rule of Direct Sum Decomposition for Substitution of Subscript Set}

Now we state the proposition:

(3) Let us consider non empty sets $I, J$, a function $a$ from $I$ into $J$, a multiplicative magma family $F$ of $J$, and an element $x$ of $\prod F$. Then $x \cdot a \in \prod(F \cdot a)$. Proof: Reconsider $y=x \cdot a$ as a many sorted set indexed by $I$. Reconsider $z=$ the support of $F \cdot a$ as a many sorted set indexed by $I$. For every object $i$ such that $i \in I$ holds $y(i) \in z(i)$ by [7, (13)].

Let $I, J$ be non empty sets, $a$ be a function from $I$ into $J$, and $F$ be a multiplicative magma family of $J$. The functor $\operatorname{Trans} \prod(F, a)$ yielding a function from $\prod F$ into $\prod(F \cdot a)$ is defined by

(Def. 2) for every element $x$ of $\prod F$, it $(x)=x \cdot a$.

Now we state the proposition:

(4) Let us consider non empty sets $I, J$, a function $a$ from $I$ into $J$, and a multiplicative magma family $F$ of $J$. Then $\operatorname{Trans} \prod(F, a)$ is multiplicative.

Proof: Reconsider $f=\operatorname{Trans} \prod(F, a)$ as a function from $\prod F$ into $\prod(F \cdot a)$. For every elements $x, y$ of $\prod F, f(x \cdot y)=f(x) \cdot f(y)$ by (3), [7, (13)], [10, (1)], [18, (27)]. 
Let $I, J$ be non empty sets, $a$ be a function from $I$ into $J$, and $F$ be a group family of $J$. Let us observe that the functor $\operatorname{Trans} \prod(F, a)$ yields a homomorphism from $\prod F$ to $\prod(F \cdot a)$. Now we state the propositions:

(5) Let us consider non empty sets $I, J$, a function $a$ from $I$ into $J$, a multiplicative magma family $F$ of $J$, and an element $y$ of $\Pi(F \cdot a)$. If $a$ is bijective, then $y \cdot a^{-1} \in \prod F$.

Proof: Set $x=y \cdot a^{-1}$. For every object $j$ such that $j \in J$ holds $x(j) \in$ (the support of $F)(j)$ by [7, (32), (13)].

(6) Let us consider non empty sets $I, J$, a function $a$ from $I$ into $J$, and functions $x, y$. Suppose $\operatorname{dom} x=I$ and $\operatorname{dom} y=J$ and $a$ is bijective. Then $x=y \cdot a$ if and only if $y=x \cdot a^{-1}$.

(7) Let us consider non empty sets $I, J$, a multiplicative magma family $F$ of $J$, and a function $a$ from $I$ into $J$. Suppose $a$ is bijective. Then

(i) $\operatorname{dom} \operatorname{Trans} \prod(F, a)=\Omega_{\prod_{F}}$, and

(ii) $\operatorname{rng} \operatorname{Trans} \prod(F, a)=\Omega \prod(F \cdot a) \cdot$

The theorem is a consequence of (5) and (6).

(8) Let us consider non empty sets $I, J$, a function $a$ from $I$ into $J$, and a multiplicative magma family $F$ of $J$. If $a$ is bijective, then $\operatorname{Trans} \prod(F, a)$ is bijective.

Proof: Reconsider $f=\operatorname{Trans} \prod(F, a)$ as a function from $\prod F$ into $\prod(F \cdot a)$. $\operatorname{dom} f=\Omega_{\prod_{F}}$ and $\operatorname{rng} f=\Omega_{\prod_{(F \cdot a)}}$. For every objects $x, y$ such that $x$, $y \in \operatorname{dom} f$ and $f(x)=f(y)$ holds $x=y$ by [7, (86)].

Let us consider non empty sets $I, J$, a function $a$ from $I$ into $J$, a group family $F$ of $J$, and a function $x$. Now we state the propositions:

(9) If $a$ is one-to-one, then $a^{\circ}(\operatorname{support}(x \cdot a, F \cdot a)) \subseteq \operatorname{support}(x, F)$.

ProOF: For every object $j$ such that $j \in a^{\circ}(\operatorname{support}(x \cdot a, F \cdot a))$ holds $j \in \operatorname{support}(x, F)$ by [7, (13)].

(10) If $a$ is onto, then $\operatorname{support}(x, F) \subseteq a^{\circ}(\operatorname{support}(x \cdot a, F \cdot a))$.

ProOF: For every object $j$ such that $j \in \operatorname{support}(x, F)$ holds

$j \in a^{\circ}(\operatorname{support}(x \cdot a, F \cdot a))$ by [8, (11)], [7, (13)].

(11) If $a$ is one-to-one, then if $x \in \operatorname{sum} F$, then $x \cdot a \in \operatorname{sum}(F \cdot a)$. The theorem is a consequence of (3) and (9).

(12) If $a$ is bijective, then $x \in \operatorname{sum} F$ iff $x \cdot a \in \operatorname{sum}(F \cdot a)$ and $\operatorname{dom} x=J$. The theorem is a consequence of (11).

Let $I, J$ be non empty sets, $a$ be a function from $I$ into $J$, and $F$ be a group family of $J$. Assume $a$ is bijective. The functor $\operatorname{Trans} \sum(F, a)$ yielding a function from $\operatorname{sum} F$ into $\operatorname{sum}(F \cdot a)$ is defined by the term 
(Def. 3) $\operatorname{Trans} \prod(F, a)\lceil\operatorname{sum} F$.

Now we state the proposition:

(13) Let us consider groups $G, H$, a subgroup $H_{0}$ of $H$, and a homomorphism $f$ from $G$ to $H$. Suppose $\operatorname{rng} f \subseteq \Omega_{H_{0}}$. Then $f$ is a homomorphism from $G$ to $H_{0}$.

ProOF: Reconsider $g=f$ as a function from $G$ into $H_{0}$. For every elements $a, b$ of $G, g(a \cdot b)=g(a) \cdot g(b)$ by [16, (43)].

Let $I, J$ be non empty sets, $a$ be a function from $I$ into $J$, and $F$ be a group family of $J$. Assume $a$ is bijective. Let us observe that the functor $\operatorname{Trans} \sum(F, a)$ yields a homomorphism from $\operatorname{sum} F$ to $\operatorname{sum}(F \cdot a)$. Now we state the propositions:

(14) Let us consider non empty sets $I, J$, a function $a$ from $I$ into $J$, and a group family $F$ of $J$. If $a$ is bijective, then $\operatorname{Trans} \sum(F, a)$ is bijective.

Proof: Reconsider $f=\operatorname{Trans} \prod(F, a)$ as a homomorphism from $\prod F$ to $\prod(F \cdot a)$. Reconsider $g=\operatorname{Trans} \sum(F, a)$ as a homomorphism from $\operatorname{sum} F$ to $\operatorname{sum}(F \cdot a) . f$ is bijective. For every object $y$ such that $y \in \Omega_{\operatorname{sum}(F \cdot a)}$ holds $y \in \operatorname{rng} g$ by [16. (42)], (5), (6), (12).

(15) Let us consider a group $G$, non empty sets $I, J$, a direct sum components $F$ of $G$ and $J$, and a function $a$ from $I$ into $J$. If $a$ is bijective, then $F \cdot a$ is a direct sum components of $G$ and $I$. The theorem is a consequence of (14).

(16) Let us consider a non empty set $I$, and a group $G$. Then every internal direct sum components of $G$ and $I$ is a subgroup family of $I$ and $G$.

(17) Let us consider non empty sets $I, J$, a group $G$, a function $x$ from $I$ into $G$, a function $y$ from $J$ into $G$, and a function $a$ from $I$ into $J$. Suppose $a$ is onto and $x=y \cdot a$. Then support $y=a^{\circ}(\operatorname{support} x)$.

(18) Let us consider non empty sets $I$, $J$, a commutative group $G$, a finitesupport function $x$ from $I$ into $G$, a finite-support function $y$ from $J$ into $G$, and a function $a$ from $I$ into $J$. If $a$ is bijective and $x=y \cdot a$, then $\prod x=\prod y$.

PROOF: Reconsider $S_{1}=\operatorname{support} x$ as a finite set. Reconsider $S_{2}=$ support $y$ as a finite set. Reconsider $s_{1}=\operatorname{CFS}\left(S_{1}\right)$ as a finite sequence of elements of $S_{1}$. Reconsider $s_{2}=\operatorname{CFS}\left(S_{2}\right)$ as a finite sequence of elements of $S_{2}$. Reconsider $x_{1}=x\left\lceil S_{1}\right.$ as a function from $S_{1}$ into $G$. Consider $x_{2}$ being a finite sequence of elements of $G$ such that $\prod x_{1}=\prod x_{2}$ and $x_{2}=x_{1} \cdot s_{1}$. Reconsider $y_{1}=y\left\lceil S_{2}\right.$ as a function from $S_{2}$ into $G$. Consider $y_{2}$ being a finite sequence of elements of $G$ such that $\prod y_{1}=\prod y_{2}$ and $y_{2}=y_{1} \cdot s_{2}$. $S_{2}=a^{\circ} S_{1} \cdot \overline{\overline{S_{1}}}=\overline{\overline{S_{2}}}$ by [1, (66)], [8, (25)], [17, (63)], [8, (17), (29)]. Reconsider $n=\overline{\overline{S_{1}}}$ as a natural number. Reconsider $a_{1}=a \uparrow S_{1}$ as a function from $S_{1}$ into $J$. Reconsider $a_{2}=s_{2}{ }^{-1}$ as a function from $S_{2}$ into $\operatorname{Seg} n$. 
Reconsider $p=a_{2} \cdot a_{1} \cdot s_{1}$ as a function. If $S_{2}$ is not empty, then $x_{2}=y_{2} \cdot p$ by [18, (27)], [7, (3), (12), (47)].

(19) Let us consider non empty sets $I, J$, a group $G$, a finite-support function $x$ from $I$ into $G$, a finite-support function $y$ from $J$ into $G$, and a function $a$ from $I$ into $J$. Suppose $a$ is bijective and $x=y \cdot a$ and for every elements $i, j$ of $I, x(i) \cdot x(j)=x(j) \cdot x(i)$. Then $\prod x=\prod y$. The theorem is a consequence of $(18)$.

(20) Let us consider a group $G$, non empty sets $I, J$, an internal direct sum components $F$ of $G$ and $J$, and a function $a$ from $I$ into $J$. Suppose $a$ is bijective. Then $F \cdot a$ is an internal direct sum components of $G$ and $I$. Proof: Reconsider $E=F \cdot a$ as a direct sum components of $G$ and $I$. For every element $i$ of $I, E(i)$ is a subgroup of $G$ by [7, (13)]. There exists a homomorphism $h$ from $\operatorname{sum} E$ to $G$ such that $h$ is bijective and for every finite-support function $x$ from $I$ into $G$ such that $x \in \operatorname{sum} E$ holds $h(x)=\prod x$ by (14), [17, (62), (63)], [12, (25)].

\section{Conservation Rule of Direct Sum Decomposition for Flattening}

Let $I$ be a non empty set and $J$ be a many sorted set indexed by $I$.

A $J$-indexed family of multiplicative magma families is a many sorted set indexed by $I$ and is defined by

(Def. 4) for every element $i$ of $I, i t(i)$ is a multiplicative magma family of $J(i)$.

A $J$-indexed family of group families is a $J$-indexed family of multiplicative magma families and is defined by

(Def. 5) for every element $i$ of $I$, it $(i)$ is a group family of $J(i)$.

Let $N$ be a $J$-indexed family of multiplicative magma families and $i$ be an element of $I$. One can verify that the functor $N(i)$ yields a multiplicative magma family of $J(i)$. Let $N$ be a $J$-indexed family of group families. Observe that the functor $N(i)$ yields a group family of $J(i)$. Let $J$ be a disjoint valued many sorted set indexed by $I$ and $F$ be a $J$-indexed family of group families. One can verify that the functor $\bigcup F$ yields a group family of $\bigcup J$. Now we state the proposition:

(21) Let us consider a non empty set $I$, a disjoint valued many sorted set $J$ indexed by $I$, a $J$-indexed family of group families $F$, an element $j$ of $I$, and an object $i$. If $i \in J(j)$, then $(\bigcup F)(i)=F(j)(i)$.

Let $I$ be a non empty set, $J$ be a many sorted set indexed by $I$, and $F$ be a $J$ indexed family of multiplicative magma families. The functor $\operatorname{ProdBundle}(F)$ yielding a multiplicative magma family of $I$ is defined by 
(Def. 6) for every element $i$ of $I$, it $(i)=\prod(F(i))$.

Let $F$ be a $J$-indexed family of group families.

Note that the functor ProdBundle $(F)$ yields a group family of $I$. The functor SumBundle $(F)$ yielding a group family of $I$ is defined by

(Def. 7) for every element $i$ of $I, i t(i)=\operatorname{sum}(F(i))$.

Let $F$ be a $J$-indexed family of multiplicative magma families. The functor $\mathrm{d} \prod F$ yielding a multiplicative magma is defined by the term

(Def. 8) ПProdBundle $(F)$.

Let $J$ be a non-empty many sorted set indexed by $I$. One can check that $\mathrm{d} \prod F$ is non empty and constituted functions.

Let $F$ be a $J$-indexed family of group families. Observe that $\mathrm{d} \prod F$ is grouplike and associative.

The functor $\mathrm{d} \sum F$ yielding a group is defined by the term

(Def. 9) sum SumBundle $(F)$.

Note that $\mathrm{d} \sum F$ is non empty and constituted functions.

Let us consider a non empty set $I$ and group families $F_{1}, F_{2}$ of $I$.

Let us assume that for every element $i$ of $I, F_{1}(i)$ is a subgroup of $F_{2}(i)$. Now we state the propositions:

(22) $\prod F_{1}$ is a subgroup of $\prod F_{2}$.

Proof: For every object $x$ such that $x \in \Omega_{\prod F_{1}}$ holds $x \in \Omega_{\prod F_{2}}$. Reconsider $f_{2}=$ (the multiplication of $\left.\prod F_{2}\right) \uparrow \Omega_{\prod F_{1}}$ as a function from $\Omega_{\prod F_{1}} \times \Omega_{\prod F_{1}}$ into $\Omega_{\prod F_{2}}$. Reconsider $f_{1}=$ the multiplication of $\prod F_{1}$ as a function from $\Omega_{\prod F_{1}} \times \Omega_{\prod F_{1}}$ into $\Omega_{\prod F_{2}}$. For every sets $x, y$ such that $x, y \in \Omega_{\prod F_{1}}$ holds $f_{1}(x, y)=f_{2}(x, y)$ by [10, (1)], [16, (43)], [7, (49)], [9, $(87)]$.

(23) $\operatorname{sum} F_{1}$ is a subgroup of $\operatorname{sum} F_{2}$.

Proof: For every object $x$ such that $x \in \Omega_{\mathrm{sum} F_{1}}$ holds $x \in \Omega_{\mathrm{sum} F_{2}}$ by [16, (40)], (22), [16, (42), (44)]. $\prod F_{1}$ is a subgroup of $\prod F_{2}$.

(24) Let us consider a non empty set $I$, a non-empty many sorted set $J$ indexed by $I$, and a $J$-indexed family of group families $F$. Then $\mathrm{d} \sum F$ is a subgroup of $\mathrm{d} \prod F$. The theorem is a consequence of (22).

Let $I$ be a non empty set, $J$ be a non-empty, disjoint valued many sorted set indexed by $I$, and $F$ be a $J$-indexed family of group families. One can verify that the functor $\mathrm{d} \sum F$ yields a subgroup of $\mathrm{d} \prod F$. The functor $\operatorname{dProd} 2 \operatorname{Prod}(F)$ yielding a homomorphism from $\mathrm{d} \prod F$ to $\Pi \bigcup F$ is defined by

(Def. 10) for every element $x$ of $\mathrm{d} \prod F$ and for every element $i$ of $I, x(i)=i t(x)\lceil J(i)$.

Now we state the proposition: 
(25) Let us consider a non empty set $I$, a non-empty, disjoint valued many sorted set $J$ indexed by $I$, a $J$-indexed family of group families $F$, an element $y$ of $\prod \cup F$, and an element $i$ of $I$. Then $y\left\lceil J(i) \in \prod(F(i))\right.$.

Proof: Set $x=y\lceil J(i)$. Set $z=$ the support of $F(i)$. For every object $j$ such that $j \in J(i)$ holds $x(j) \in z(j)$ by [7, (49), (1)].

Let $I$ be a non empty set, $J$ be a non-empty, disjoint valued many sorted set indexed by $I$, and $F$ be a $J$-indexed family of group families. Note that $\mathrm{dProd} 2 \operatorname{Prod}(F)$ is bijective.

The functor $\operatorname{Prod} 2 \mathrm{dProd}(F)$ yielding a homomorphism from $\prod \cup F$ to $\mathrm{d} \prod F$ is defined by the term

(Def. 11) (dProd2Prod $(F))^{-1}$.

Now we state the proposition:

(26) Let us consider a non empty set $I$, a non-empty, disjoint valued many sorted set $J$ indexed by $I$, a $J$-indexed family of group families $F$, an element $x$ of $\prod \cup F$, and an element $i$ of $I$. Then $x\lceil J(i)=(\operatorname{Prod} 2 \mathrm{dProd}(F))(x)(i)$.

Let $I$ be a non empty set, $J$ be a non-empty, disjoint valued many sorted set indexed by $I$, and $F$ be a $J$-indexed family of group families. Note that $\operatorname{Prod} 2 \mathrm{dProd}(F)$ is bijective.

(27) Let us consider a non empty set $I$, a non-empty, disjoint valued many sorted set $J$ indexed by $I$, and a $J$-indexed family of group families $F$. Then $\operatorname{Prod} 2 \mathrm{dProd}(F)=(\operatorname{dProd} 2 \operatorname{Prod}(F))^{-1}$.

Let $I$ be a non empty set, $J$ be a non-empty, disjoint valued many sorted set indexed by $I, F$ be a $J$-indexed family of group families, and $x$ be a function. The functor $\operatorname{rsupport}(x, F)$ yielding a disjoint valued many sorted set indexed by $I$ is defined by

(Def. 12) for every element $i$ of $I, i t(i)=\operatorname{support}(x\lceil J(i), F(i))$.

Now we state the propositions:

(28) Let us consider a non empty set $I$, a non-empty, disjoint valued many sorted set $J$ indexed by $I$, a $J$-indexed family of group families $F$, and a function $x$. Then $\operatorname{support}(x, \bigcup F)=\bigcup \operatorname{rsupport}(x, F)$.

Proof: Set $y=\operatorname{rsupport}(x, F)$. For every object $j, j \in \operatorname{support}(x, \bigcup F)$ iff $j \in \bigcup y$ by (21), [7, (49), (3)], [9, (74)].

(29) Let us consider a non empty set $I$, a non-empty, disjoint valued many sorted set $J$ indexed by $I$, a $J$-indexed family of group families $F$, and functions $x, y, z$. Suppose $z \in \mathrm{d} \prod F$ and $x=(\operatorname{dProd} 2 \operatorname{Prod}(F))(z)$. Then

(i) $\operatorname{rsupport}(x, F)\lceil\operatorname{support}(z, \operatorname{SumBundle}(F))$ is a non-empty, disjoint valued many sorted set indexed by $\operatorname{support}(z, \operatorname{SumBundle}(F))$, and

(ii) $\operatorname{support}(x, \bigcup F)=\bigcup(\operatorname{rsupport}(x, F)\lceil\operatorname{support}(z, \operatorname{SumBundle}(F)))$. 
Proof: Set $s_{1}=\operatorname{rsupport}(x, F)$. Set $s_{2}=\operatorname{support}(z, \operatorname{SumBundle}(F))$. Set $f=s_{1}\left\lceil s_{2}\right.$. For every objects $s, t$ such that $s \neq t$ holds $f(s)$ misses $f(t)$ by [7, (47)]. $\emptyset \notin \operatorname{rng} f$ by [7, (47)], [10, (5)], [16, (44)]. $\operatorname{support}(x, \bigcup F)=\bigcup s_{1}$. For every object $k$ such that $k \in \operatorname{support}(x, \bigcup F)$ holds $k \in \bigcup\left(s_{1}\left\lceil s_{2}\right)\right.$ by [10, (6)], [16, (44)], [18, (57)], [7, (47), (3)].

(30) Let us consider a non empty set $I$, a non-empty, disjoint valued many sorted set $J$ indexed by $I$, a $J$-indexed family of group families $F$, and a function $y$. Suppose $y \in \operatorname{sum} \bigcup F$. Then there exists a function $x$ such that

(i) $y=(\mathrm{dProd} 2 \operatorname{Prod}(F))(x)$, and

(ii) $x \in \mathrm{d} \sum F$.

Proof: Consider $x$ being an element of $\Omega_{\mathrm{d}} \prod_{F}$ such that $y=($ dProd2Prod $(F))(x)$. Set $s_{1}=\operatorname{rsupport}(y, F)$. $\operatorname{support}(y, \bigcup F)=\bigcup s_{1}$. For every element $i$ of $I, x(i) \in(\operatorname{SumBundle}(F))(i)$ by [7, (3)], [9, (74)], [12, (8)]. Set $S=\operatorname{SumBundle}(F)$. Reconsider $W=$ the support of $S$ as a many sorted set indexed by $I$. For every object $i$ such that $i \in I$ holds $x(i) \in W(i)$. Reconsider $s_{2}=s_{1} \uparrow \operatorname{support}(x, \operatorname{SumBundle}(F))$ as a non-empty, disjoint valued many sorted set indexed by $\operatorname{support}(x, \operatorname{SumBundle}(F))$. $\bigcup s_{2}$ is finite. $\operatorname{dom} s_{2}$ is finite.

(31) Let us consider a non empty set $I$, a non-empty, disjoint valued many sorted set $J$ indexed by $I$, a $J$-indexed family of group families $F$, and functions $x, y$. Suppose $x, x \in \mathrm{d} \sum F$. Then $(\operatorname{dProd} 2 \operatorname{Prod}(F))(x) \in \operatorname{sum} \bigcup F$. Proof: Reconsider $y=(\operatorname{dProd} 2 \operatorname{Prod}(F))(x)$ as an element of $\prod \cup F$. Set $s_{1}=\operatorname{rsupport}(y, F)$. Reconsider $s_{2}=s_{1}\lceil\operatorname{support}(x, \operatorname{SumBundle}(F))$ as a non-empty, disjoint valued many sorted set indexed by $\operatorname{support}(x, \operatorname{SumBundle}(F))$. For every object $i$ such that $i \in \operatorname{dom} s_{2}$ holds $s_{2}(i)$ is finite by [16, (40)], [7, (49)]. $\operatorname{support}(y, \bigcup F)$ is finite.

(32) Let us consider a non empty set $I$, a non-empty, disjoint valued many sorted set $J$ indexed by $I$, and a $J$-indexed family of group families $F$. Then $\operatorname{rng}\left(\operatorname{dProd} 2 \operatorname{Prod}(F)\left\lceil\mathrm{d} \sum F\right)=\Omega_{\text {sum } \bigcup F}\right.$.

Proof: For every object $y, y \in \operatorname{rng}\left(\operatorname{dProd} 2 \operatorname{Prod}(F)\left\lceil\Omega_{\mathrm{d} \sum F}\right)\right.$ iff $y \in$ $\Omega_{\text {sum } \bigcup F}$ by [18, (61)], (31), [7, (47)], (30).

Let $I$ be a non empty set, $J$ be a non-empty, disjoint valued many sorted set indexed by $I$, and $F$ be a $J$-indexed family of group families. The functor $\mathrm{dSum} 2 \operatorname{Sum}(F)$ yielding a homomorphism from $\mathrm{d} \sum F$ to $\operatorname{sum} \bigcup F$ is defined by the term

(Def. 13) $\operatorname{dProd} 2 \operatorname{Prod}(F)\left\lceil\mathrm{d} \sum F\right.$.

One can verify that $\mathrm{dSum} 2 \mathrm{Sum}(F)$ is bijective. 
The functor $\operatorname{Sum} 2 \mathrm{dSum}(F)$ yielding a homomorphism from $\operatorname{sum} \bigcup F$ to $\mathrm{d} \sum F$ is defined by the term

(Def. 14) (dSum2Sum $(F))^{-1}$.

Now we state the proposition:

(33) Let us consider a non empty set $I$, a non-empty, disjoint valued many sorted set $J$ indexed by $I$, and a $J$-indexed family of group families $F$. Then $\operatorname{Sum} 2 \mathrm{~d} \operatorname{Sum}(F)=\operatorname{Prod} 2 \mathrm{dProd}(F)\lceil\operatorname{sum} \bigcup F$. The theorem is a consequence of $(2)$.

Let $I$ be a non empty set, $J$ be a non-empty, disjoint valued many sorted set indexed by $I$, and $F$ be a $J$-indexed family of group families. One can check that $\operatorname{Sum} 2 \mathrm{~d} \operatorname{Sum}(F)$ is bijective.

Now we state the proposition:

(34) Let us consider a non empty set $I$, a non-empty, disjoint valued many sorted set $J$ indexed by $I$, and a $J$-indexed family of group families $F$. Then $\operatorname{dSum} 2 \operatorname{Sum}(F)=(\operatorname{Sum} 2 \mathrm{dSum}(F))^{-1}$.

Let $I$ be a non empty set, $G$ be a group, and $F$ be an internal direct sum components of $G$ and $I$. The functor $\operatorname{InterHom}(F)$ yielding a homomorphism from $\operatorname{sum} F$ to $G$ is defined by

(Def. 15) it is bijective and for every finite-support function $x$ from $I$ into $G$ such that $x \in \operatorname{sum} F$ holds it $(x)=\prod x$.

Let $J$ be a non-empty, disjoint valued many sorted set indexed by $I, M$ be a direct sum components of $G$ and $I, N$ be a $J$-indexed family of group families, and $h$ be a many sorted set indexed by $I$. Assume for every element $i$ of $I$, there exists a homomorphism $h_{0}$ from $(\operatorname{SumBundle}(N))(i)$ to $M(i)$ such that $h_{0}=h(i)$ and $h_{0}$ is bijective. The functor $\operatorname{ProdHom}(G, M, N, h)$ yielding a homomorphism from $\mathrm{d} \sum N$ to $\operatorname{sum} M$ is defined by

(Def. 16) $i t=\operatorname{SumMap}(\operatorname{SumBundle}(N), M, h)$ and it is bijective.

Now we state the propositions:

(35) Let us consider a non empty set $I$, a non-empty, disjoint valued many sorted set $J$ indexed by $I$, a group $G$, a direct sum components $M$ of $G$ and $I$, and a $J$-indexed family of group families $N$. Suppose for every element $i$ of $I, N(i)$ is a direct sum components of $M(i)$ and $J(i)$. Then $\cup N$ is a direct sum components of $G$ and $\bigcup J$.

ProOF: Consider $f_{2}$ being a homomorphism from sum $M$ to $G$ such that $f_{2}$ is bijective. Define $\mathcal{P}$ (object) $=\Omega_{\text {sum }\left(N\left(\$_{1}(\in I)\right)\right)}$. Consider $D_{2}$ being a function such that dom $D_{2}=I$ and for every object $i$ such that $i \in I$ holds $D_{2}(i)=\mathcal{P}(i)$ from [7, Sch. 3]. Define $\mathcal{Q}$ (object) $=\Omega_{M\left(\$_{1}(\in I)\right)}$. Consider $R_{1}$ being a function such that $\operatorname{dom} R_{1}=I$ and for every object $i$ such 
that $i \in I$ holds $R_{1}(i)=\mathcal{Q}(i)$ from [7, Sch. 3]. Define $\mathcal{R}$ [object, object] $\equiv$ there exists a homomorphism $f_{3}$ from $\operatorname{sum}\left(N\left(\$_{1}(\in I)\right)\right)$ to $M\left(\$_{1}(\in I)\right)$ such that $f_{3}=\$_{2}$ and $f_{3}$ is bijective. For every element $i$ of $I$, there exists an element $y$ of $\bigcup D_{2} \dot{\rightarrow} \bigcup R_{1}$ such that $\mathcal{R}[i, y]$ by [7, (3)], [9, (74)]. Consider $f_{1}$ being a function from $I$ into $\bigcup D_{2} \dot{\rightarrow} \bigcup R_{1}$ such that for every element $i$ of $I, \mathcal{R}\left[i, f_{1}(i)\right]$ from [8, Sch. 3]. For every element $i$ of $I$, there exists a homomorphism $h_{0}$ from (SumBundle $\left.(N)\right)(i)$ to $M(i)$ such that $h_{0}=f_{1}(i)$ and $h_{0}$ is bijective.

(36) Let us consider a non empty set $I$, a non-empty, disjoint valued many sorted set $J$ indexed by $I$, a group $G$, an internal direct sum components $M$ of $G$ and $I$, and a $J$-indexed family of group families $N$. Suppose for every element $i$ of $I, N(i)$ is an internal direct sum components of $M(i)$ and $J(i)$. Then $\bigcup N$ is an internal direct sum components of $G$ and $\bigcup J$. Proof: Consider $f_{3}$ being a homomorphism from sum $M$ to $G$ such that $f_{3}$ is bijective and for every finite-support function $x$ from $I$ into $G$ such that $x \in \operatorname{sum} M$ holds $f_{3}(x)=\prod x$. Define $\mathcal{Q}[$ object, object $] \equiv$ there exists an internal direct sum components $N_{1}$ of $M\left(\$_{1}(\in I)\right)$ and $J\left(\$_{1}(\in I)\right)$ such that $N_{1}=N\left(\$_{1}\right)$ and $\$_{2}=\operatorname{InterHom}\left(N_{1}\right)$. For every object $x$ such that $x \in I$ there exists an object $y$ such that $\mathcal{Q}[x, y]$. Consider $f_{1}$ being a function such that $\operatorname{dom} f_{1}=I$ and for every object $i$ such that $i \in I$ holds $\mathcal{Q}\left[i, f_{1}(i)\right]$ from [7, Sch. 2]. Set $f_{2}=\operatorname{ProdHom}\left(G, M, N, f_{1}\right)$. For every element $i$ of $I$, there exists a homomorphism $h_{0}$ from $(\operatorname{SumBundle}(N))(i)$ to $M(i)$ such that $h_{0}=f_{1}(i)$ and $h_{0}$ is bijective and for every finite-support function $x$ from $J(i)$ into $M(i)$ such that $x \in(\operatorname{SumBundle}(N))(i)$ holds $h_{0}(x)=\prod x$. For every element $i$ of $I$, there exists a homomorphism $h_{0}$ from (SumBundle $(N))(i)$ to $M(i)$ such that $h_{0}=f_{1}(i)$ and $h_{0}$ is bijective. Reconsider $h=f_{3} \cdot f_{2} \cdot \operatorname{Sum} 2 \mathrm{dSum}(N)$ as a homomorphism from sum $\bigcup N$ to $G$. Reconsider $U_{2}=\bigcup J$ as a non empty set. Reconsider $U_{3}=\bigcup N$ as a direct sum components of $G$ and $U_{2}$. For every object $j$ such that $j \in U_{2}$ holds $U_{3}(j)$ is a subgroup of $G$ by (21), [16, (56)]. For every finite-support function $x$ from $U_{2}$ into $G$ such that $x \in \operatorname{sum} U_{3}$ holds $h(x)=\prod x$ by [16, (42), (40)], [7, (13)], [8, (5), (15)].

\section{Conservation Rule of Direct Sum Decomposition for Layering}

Now we state the propositions:

(37) Let us consider a non empty set $I$, a non-empty, disjoint valued many sorted set $J$ indexed by $I$, a group $G$, a group family $M$ of $I$, and a $J$-indexed family of group families $N$. Suppose $\bigcup N$ is a direct sum components of $G$ and $\bigcup J$ and for every element $i$ of $I, N(i)$ is a direct sum 
components of $M(i)$ and $J(i)$. Then $M$ is a direct sum components of $G$ and $I$.

Proof: Set $U_{3}=\bigcup N$. Consider $f_{4}$ being a homomorphism from sum $U_{3}$ to $G$ such that $f_{4}$ is bijective. Define $\mathcal{P}$ (object) $=$ the carrier of $\operatorname{sum}\left(N\left(\$_{1}(\epsilon\right.\right.$ $I))$ ). Consider $D_{2}$ being a function such that $\operatorname{dom} D_{2}=I$ and for every object $i$ such that $i \in I$ holds $D_{2}(i)=\mathcal{P}(i)$ from [7, Sch. 3]. Define $\mathcal{Q}($ object $)=$ the carrier of $M\left(\$_{1}(\in I)\right)$. Consider $R_{1}$ being a function such that $\operatorname{dom} R_{1}=I$ and for every object $i$ such that $i \in I$ holds $R_{1}(i)=\mathcal{Q}(i)$ from [7, Sch. 3]. Define $\mathcal{R}$ [object, object] $\equiv$ there exists a homomorphism $f_{3}$ from $M\left(\$_{1}(\in I)\right)$ to $\operatorname{sum}\left(N\left(\$_{1}(\in I)\right)\right)$ such that $f_{3}=\$_{2}$ and $f_{3}$ is bijective. For every element $i$ of $I$, there exists an element $y$ of $\bigcup R_{1} \dot{\cup} \cup D_{2}$ such that $\mathcal{R}[i, y]$ by [17, (62), (63)], [7, (3)], [9, (74)]. Consider $f_{1}$ being a function from $I$ into $\bigcup R_{1} \dot{\rightarrow} \bigcup D_{2}$ such that for every element $i$ of $I$, $\mathcal{R}\left[i, f_{1}(i)\right]$ from [8, Sch. 3]. For every element $i$ of $I$, there exists a homomorphism $h_{0}$ from $M(i)$ to $(\operatorname{SumBundle}(N))(i)$ such that $h_{0}=f_{1}(i)$ and $h_{0}$ is bijective.

(38) Let us consider a non empty set $I$, a non-empty, disjoint valued many sorted set $J$ indexed by $I$, a group $G$, a subgroup family $M$ of $I$ and $G$, and a $J$-indexed family of group families $N$. Suppose $\bigcup N$ is an internal direct sum components of $G$ and $\bigcup J$ and for every element $i$ of $I, N(i)$ is an internal direct sum components of $M(i)$ and $J(i)$. Then $M$ is an internal direct sum components of $G$ and $I$.

ProOF: Reconsider $U_{2}=\bigcup J$ as a non empty set. Consider $f_{4}$ being a homomorphism from sum $\bigcup N$ to $G$ such that $f_{4}$ is bijective and for every finite-support function $x$ from $U_{2}$ into $G$ such that $x \in \operatorname{sum} \cup N$ holds $f_{4}(x)=\prod x$. Define $\mathcal{Q}[$ object, object $] \equiv$ there exists an internal direct sum components $N_{1}$ of $M\left(\$_{1}(\in I)\right)$ and $J\left(\$_{1}(\in I)\right)$ such that $N_{1}=N\left(\$_{1}\right)$ and $\$_{2}=\left(\operatorname{InterHom}\left(N_{1}\right)\right)^{-1}$. For every object $x$ such that $x \in I$ there exists an object $y$ such that $\mathcal{Q}[x, y]$.

Consider $f_{1}$ being a function such that dom $f_{1}=I$ and for every object $i$ such that $i \in I$ holds $\mathcal{Q}\left[i, f_{1}(i)\right]$ from [7, Sch. 2]. Reconsider $f_{3}=\operatorname{SumMap}\left(M,(\operatorname{SumBundle}(N)), f_{1}\right)$ as a homomorphism from $\operatorname{sum} M$ to $\mathrm{d} \sum N$. For every element $i$ of $I$, there exists a homomorphism $h_{0}$ from $M(i)$ to $(\operatorname{SumBundle}(N))(i)$ such that $h_{0}=f_{1}(i)$ and $h_{0}$ is bijective by [17, (62), (63)]. Reconsider $h=f_{4} \cdot \mathrm{d} \operatorname{Sum} 2 \operatorname{Sum}(N) \cdot f_{3}$ as a homomorphism from sum $M$ to $G$. For every element $i$ of $I$, there exists a homomorphism $h_{0}$ from $(\operatorname{SumBundle}(N))(i)$ to $M(i)$ such that $h_{0}^{-1}=f_{1}(i)$ and $h_{0}$ is bijective and for every finite-support function $x$ from $J(i)$ into $M(i)$ such that $x \in(\operatorname{SumBundle}(N))(i)$ holds $h_{0}(x)=\prod x$. For every element $i$ of $I$, there exists a homomorphism $h_{0}$ from $(\operatorname{SumBundle}(N))(i)$ to $M(i)$ such 
that $h_{0}^{-1}=f_{1}(i)$ and $h_{0}$ is bijective. For every finite-support function $x$ from $I$ into $G$ such that $x \in \operatorname{sum} M$ holds $h(x)=\prod x$ by [16, (40)], [7, (13)], [8, (5), (15)].

(39) Let us consider a non empty set $I_{2}$, and a group family $F_{2}$ of $I_{2}$. Suppose for every element $i$ of $I_{2}, \overline{\overline{F_{2}(i)}}=1$. Then $\overline{\bar{\alpha}}=1$, where $\alpha$ is the carrier of $\operatorname{sum} F_{2}$.

Proof: For every object $x$ such that $x \in \Omega_{\mathrm{sum} F_{2}}$ holds $x=\mathbf{1}_{\text {sum } F_{2}}$ by [16, (42)], [1, (30)], [2, (102)], [10, (5)].

(40) Let us consider a non empty set $I$, a group $G$, and a finite-support function $x$ from $I$ into $G$. Suppose for every object $i$ such that $i \in I$ holds $x(i)=\mathbf{1}_{G}$. Then $\prod x=\mathbf{1}_{G}$.

(41) Let us consider a non empty set $I$, a group $G$, a finite-support function $x$ from $I$ into $G$, and an element $a$ of $G$. If $I=\{1,2\}$ and $x=\left\langle a, \mathbf{1}_{G}\right\rangle$, then $\prod x=a$.

Proof: Reconsider $i_{1}=1$ as an element of $I$. Set $y=\left(I \longmapsto \mathbf{1}_{G}\right)+\cdot\left(i_{1}, a\right)$. For every object $i$ such that $i \in \operatorname{dom} x \operatorname{holds} x(i)=y(i)$ by [3, (44)], [4, (31), (32)], [15, (7)].

(42) Let us consider a group $G$, non empty sets $I_{1}, I_{2}$, a direct sum components $F_{1}$ of $G$ and $I_{1}$, and a group family $F_{2}$ of $I_{2}$. Suppose $I_{1}$ misses $I_{2}$ and for every element $i$ of $I_{2}, \overline{\overline{F_{2}(i)}}=1$. Then $F_{1}+F_{2}$ is a direct sum components of $G$ and $I_{1} \cup I_{2}$.

Proof: Reconsider $I=\{1,2\}$ as a non empty set. Set $J=\left\{\left\langle 1, I_{1}\right\rangle,\langle 2\right.$, $\left.\left.I_{2}\right\rangle\right\}$. For every objects $x, y_{1}, y_{2}$ such that $\left\langle x, y_{1}\right\rangle,\left\langle x, y_{2}\right\rangle \in J$ holds $y_{1}=y_{2} . \emptyset \notin \operatorname{rng} J$. For every objects $i, j$ such that $i \neq j$ holds $J(i)$ misses $J(j)$. Reconsider $M=\left\langle\operatorname{sum} F_{1}, \operatorname{sum} F_{2}\right\rangle$ as a group family of $I$. $\overline{\overline{\Omega_{\text {sum } F_{2}}}}=1$. Consider $w$ being an object such that $\{w\}=\Omega_{\mathrm{sum} F_{2}}$. For every functions $x, y$ such that $x, y \in \Omega \prod_{M}$ and $x(1)=y(1)$ holds $x=y$ by [12, (5)], [3, (44)].

Consider $h_{1}$ being a homomorphism from sum $F_{1}$ to $G$ such that $h_{1}$ is bijective. Set $C_{1}=$ the carrier of $\prod M$. Set $C_{2}=$ the carrier of $G$. Define $\mathcal{P}$ [element of $C_{1}$, element of $\left.C_{2}\right] \equiv \$_{2}=h_{1}\left(\$_{1}(1)\right)$. For every element $x$ of $C_{1}$, there exists an element $y$ of $C_{2}$ such that $\mathcal{P}[x, y]$ by [12, (5)], [3, (44)], [8, (5)]. Consider $h$ being a function from $C_{1}$ into $C_{2}$ such that for every element $x$ of $C_{1}, \mathcal{P}[x, h(x)]$ from [8, Sch. 3]. For every objects $x_{1}, x_{2}$ such that $x_{1}, x_{2} \in C_{1}$ and $h\left(x_{1}\right)=h\left(x_{2}\right)$ holds $x_{1}=x_{2}$ by [12, (5)], [3, (44)], [8, (19)]. For every object $y$ such that $y \in C_{2}$ there exists an object $x$ such that $x \in C_{1}$ and $y=h(x)$ by [8, (11)], [3, (44)]. For every elements $a, b$ of $C_{1}, h(a \cdot b)=h(a) \cdot h(b)$ by [3, (44)], [12, (5)], [10, (1)]. Reconsider $M=\left\langle\operatorname{sum} F_{1}, \operatorname{sum} F_{2}\right\rangle$ as a direct sum components 
of $G$ and $I$. Set $N=\left\langle F_{1}, F_{2}\right\rangle$. For every element $i$ of $I, N(i)$ is a group family of $J(i)$ by [3, (44)]. For every element $i$ of $I, N(i)$ is a direct sum components of $M(i)$ and $J(i)$ by [3, (44)]. For every object $x$ such that $x \in \operatorname{dom} F_{1} \cup \operatorname{dom} F_{2}$ holds if $x \in \operatorname{dom} F_{2}$, then $(\cup N)(x)=F_{2}(x)$ and if $x \notin \operatorname{dom} F_{2}$, then $(\bigcup N)(x)=F_{1}(x)$ by $(21),[3$, (44)].

(43) Let us consider a group $G$, non empty sets $I_{1}, I_{2}$, an internal direct sum components $F_{1}$ of $G$ and $I_{1}$, and a subgroup family $F_{2}$ of $I_{2}$ and $G$. Suppose $I_{1}$ misses $I_{2}$ and $F_{2}=I_{2} \longmapsto\{\mathbf{1}\}_{G}$. Then $F_{1}+\cdot F_{2}$ is an internal direct sum components of $G$ and $I_{1} \cup I_{2}$.

Proof: Reconsider $I=\{1,2\}$ as a non empty set. Set $J=\left\{\left\langle 1, I_{1}\right\rangle,\langle 2\right.$, $\left.\left.I_{2}\right\rangle\right\}$. For every objects $x, y_{1}, y_{2}$ such that $\left\langle x, y_{1}\right\rangle,\left\langle x, y_{2}\right\rangle \in J$ holds $y_{1}=y_{2} . \emptyset \notin \operatorname{rng} J$. For every objects $i, j$ such that $i \neq j$ holds $J(i)$ misses $J(j)$. Reconsider $M=\left\langle G,\{\mathbf{1}\}_{G}\right\rangle$ as a group family of $I$. For every functions $x, y$ such that $x, y \in \Omega_{M}$ and $x(1)=y(1)$ holds $x=y$ by [12, (5)], [3, (44)]. Set $h_{1}=\operatorname{id}_{(\text {the carrier of } G)}$. Set $C_{1}=$ the carrier of $\prod M$. Set $C_{2}=$ the carrier of $G$. Define $\mathcal{P}$ [element of $C_{1}$, element of $\left.C_{2}\right] \equiv$ $\$_{2}=h_{1}\left(\$_{1}(1)\right)$. For every element $x$ of $C_{1}$, there exists an element $y$ of $C_{2}$ such that $\mathcal{P}[x, y]$ by [12, (5)], [3, (44)], [8, (5)]. Consider $h$ being a function from $C_{1}$ into $C_{2}$ such that for every element $x$ of $C_{1}, \mathcal{P}[x, h(x)]$ from [8, Sch. 3]. For every objects $x_{1}, x_{2}$ such that $x_{1}, x_{2} \in C_{1}$ and $h\left(x_{1}\right)=h\left(x_{2}\right)$ holds $x_{1}=x_{2}$ by [12, (5)], [3, (44)], [8, (19)]. For every object $y$ such that $y \in C_{2}$ there exists an object $x$ such that $x \in C_{1}$ and $y=h(x)$ by [8, (11)], [3. (44)]. For every elements $a, b$ of $C_{1}, h(a \cdot b)=h(a) \cdot h(b)$ by [3, (44)], [12, (5)], [10, (1)].

Reconsider $M=\left\langle G,\{\mathbf{1}\}_{G}\right\rangle$ as a direct sum components of $G$ and $I$. For every element $i$ of $I, M(i)$ is a subgroup of $G$ by [3, (44)], [16, (54)]. For every finite-support function $x$ from $I$ into $G$ such that $x \in \operatorname{sum} M$ holds $h(x)=\prod x$ by [10, (9)], [3, (44)], (41). Set $N=\left\langle F_{1}, F_{2}\right\rangle$. For every element $i$ of $I, N(i)$ is a group family of $J(i)$ by [3, (44)]. For every element $i$ of $I$, $N(i)$ is an internal direct sum components of $M(i)$ and $J(i)$ by [3, (44)], [15, (7)], [1, (30)], (39). For every object $x$ such that $x \in \operatorname{dom} F_{1} \cup \operatorname{dom} F_{2}$ holds if $x \in \operatorname{dom} F_{2}$, then $(\bigcup N)(x)=F_{2}(x)$ and if $x \notin \operatorname{dom} F_{2}$, then $(\bigcup N)(x)=F_{1}(x)$ by $(21),[3$, (44)].

\section{REFERENCES}

[1] Grzegorz Bancerek. Cardinal numbers Formalized Mathematics, 1(2):377-382, 1990.

[2] Grzegorz Bancerek. Cardinal arithmetics Formalized Mathematics, 1(3):543-547, 1990.

[3] Grzegorz Bancerek and Krzysztof Hryniewiecki. Segments of natural numbers and finite sequences. Formalized Mathematics, 1(1):107-114, 1990.

[4] Grzegorz Bancerek and Andrzej Trybulec. Miscellaneous facts about functions Formalized 
Mathematics, 5(4):485-492, 1996.

[5] Grzegorz Bancerek, Czesław Byliński, Adam Grabowski, Artur Korniłowicz, Roman Matuszewski, Adam Naumowicz, Karol Pąk, and Josef Urban. Mizar: State-of-the-art and beyond. In Manfred Kerber, Jacques Carette, Cezary Kaliszyk, Florian Rabe, and Volker Sorge, editors, Intelligent Computer Mathematics, volume 9150 of Lecture Notes in Computer Science, pages 261-279. Springer International Publishing, 2015. ISBN 978-3319-20614-1. doi $10.1007 / 978-3-319-20615-8 \_17$.

[6] Nicolas Bourbaki. Elements of Mathematics. Algebra I. Chapters 1-3. Springer-Verlag, Berlin, Heidelberg, New York, London, Paris, Tokyo, 1989.

[7] Czesław Byliński. Functions and their basic properties Formalized Mathematics, 1(1): 55-65, 1990.

[8] Czesław Byliński. Functions from a set to a set Formalized Mathematics, 1(1):153-164, 1990.

[9] Czesław Byliński. Some basic properties of sets Formalized Mathematics, 1(1):47-53, 1990.

[10] Artur Korniłowicz. The product of the families of the groups Formalized Mathematics, 7(1):127-134, 1998.

[11] Serge Lang. Algebra. Springer, 3rd edition, 2005.

[12] Kazuhisa Nakasho, Hiroshi Yamazaki, Hiroyuki Okazaki, and Yasunari Shidama. Definition and properties of direct sum decomposition of groups. Formalized Mathematics, 23 (1):15-27, 2015. doi 10.2478/forma-2015-0002

[13] D. Robinson. A Course in the Theory of Groups. Springer New York, 2012.

[14] J.J. Rotman. An Introduction to the Theory of Groups. Springer, 1995.

[15] Andrzej Trybulec. Binary operations applied to functions Formalized Mathematics, 1 (2):329-334, 1990.

[16] Wojciech A. Trybulec. Subgroup and cosets of subgroups Formalized Mathematics, 1(5): 855-864, 1990.

[17] Woiciech A. Trybulec and Michał J. Trybulec. Homomorphisms and isomorphisms of groups. Quotient group Formalized Mathematics, 2(4):573-578, 1991.

[18] Edmund Woronowicz. Relations and their basic properties. Formalized Mathematics, 1 (1):73-83, 1990 .

Received December 31, 2015 